\title{
Experimental Study of Inconel 718 Surface Treatment by Edge Robotic Deburring with Force Control
}

\author{
A. Burghardt, ${ }^{a}$ D. Szybicki, ${ }^{a}$ K. Kurc, ${ }^{a}$ M. Muszyńska, ${ }^{a}$ and J. Mucha ${ }^{b, 1}$ \\ ${ }^{a}$ Department of Applied Mechanics and Robotics, Rzeszów University of Technology, Rzeszów, \\ Poland \\ ${ }^{b}$ Department of Mechanical Engineering, Rzeszów University of Technology, Rzeszów, Poland \\ ${ }^{1}$ j_mucha@prz.edu.pl
}

We present the results of investigations into the application of robotics for deburring and chamfering to a predefined geometric quality. The robotic application was used for a part of the manufacturing process of an aircraft engine detail. Aircraft engine diffuser machining requires manual deburring of many edges. Finishing by hand results in several non-conforming quality details for each diffuser. This paper presents the concept of edge deburring with a controlled force progression pneumatic tool. A specific methodology was used to select and optimise the edge deburring process for robotic chamfering processing to a finer machining tolerance. The investigated machining process included a measurement system for the determination of the manufactured chamfer as a function of contact forces with feed force progression. The investigation work discussed in the paper helped to identify a specific interval of processing parameters, including the contact force and TCP motion velocity at which deburring is effective and a chamfer with specific geometric tolerance is produced. The experimental part of the investigation was conducted at a preset feed force of the high-speed machining file, tool ref. FDB150. The experimental machining sample was made from poorly machinable titanium alloy (Inconel 718), a material applied in the aerospace industry. The machining process optimisation included an approximation of the chamfer width definition points. The resulting function provided a derivative, defining the chamfer value change rate and corresponding to the actual machining tool infeed. The experimental measurement results were compared to the assumed quality indicators, by which a group of suboptimal parameters was defined.

Keywords: process automation, industrial manipulators, chamfering the edges, optimization process, design for sustainability.

Introduction. Aside from the increasing popularity of CNC machine tool applications in the aerospace industry, robots have been used to replace human operators in certain manufacturing processes. There has been a noticeable trend in smart manufacturing through the application of industrial robotics [1-4]. Adaptive force control (AFC) systems are used for machining complex geometrical features, as in the case of milling [5-7]. Zuperl et al. [8] presented a method for AFC application in three-axis milling with a CNC machine tool. Optimizing the AFC CNC milling parameters facilitates a precise quality control of the geometrical structure of the surface [9]. Khanghah et al. [10] presented a study of burr removal through the application of an AFC algorithm.

Robots are not exclusively used for typical machining operations. [7] discusses a method for generating robotic motion trajectories with contact force to remove the effects of changes in processed part geometry. [11] discusses aircraft part surface finishing with a robotic manipulator programmed to polish a curved canopy of an unknown geometry. Jamisol et al. [11] provided a process control solution with a constant normal force applied to a sheet surface during tool motion with tangent contact. $[12,13]$ presents the diagnostics of a manipulator tool during a force control deburring process. Doulgeri and Karayiannidis [14] prepared one of the very few papers to date concerning a mathematical control model for force control machining of an unknown rigid convex surface. The authors of [14] proposed a control method which guarantees asymptotic stability of the force error when 
the maximum surface orientation error is less than 90 degrees. This approach ensures a defined shape error in the processing of unknown surfaces. Doulgeri et al. proposed an AFC for processing of surfaces with unknown rigidity [15]. The authors proved that the application of force control is reasonable when the estimated surface orientation matches the actual surface values. Constant working parameters, including feed force, is critical in typical manufacturing processes and other applications, including medicine [16].

High-speed drive units are used to increase machining throughput. Machining with high-speed drive units helps retain proper processing efficiency and increase product precision. There is little information concerning AFC applications in robotics for the minimization of shape errors in HSM. Matsuoka et al. [17] presented an integration of an industrial robot with a high-speed drive unit. Abele et al. [18] provided analytical modeling of this application study.

This paper presents the experimental results of robotized Inconel 718 edge milling. The paper describes the method for selecting the parameters for robotic edge deburring with an HSM file and pneumatic feed force progression.

Methodology and Results. The topic examined herein is the research and experimental work into robotic applications for aircraft component manufacturing which is epitomized by the machining of V2500 engine diffuser edges (Fig. 1a). The V2500 engine is a propulsion unit dedicated to the Airbus A320 and McDonnell Douglas MD-90 aircraft. The manufacturing operations of machining V2500 engine diffuser castings (Fig. 1b) were studied for the viability of automation. Edge deburring is one of the final manufacturing stages for these parts.

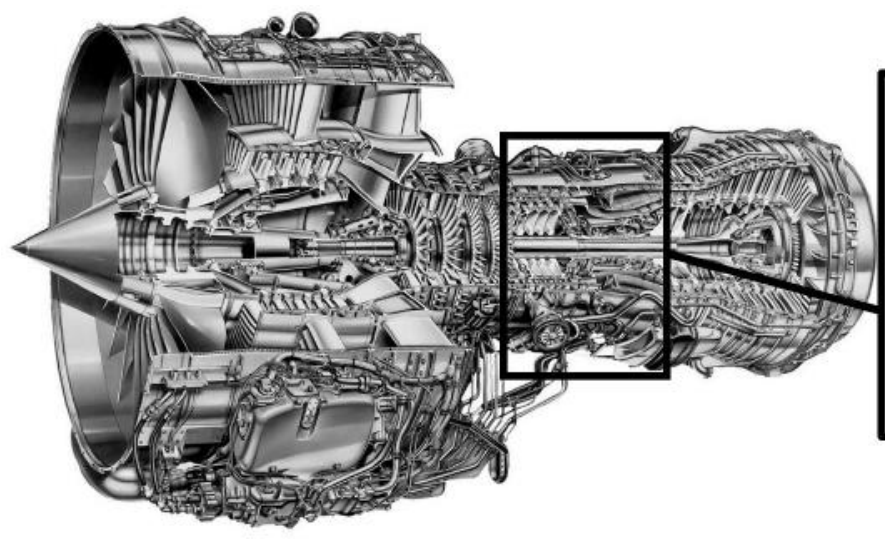

a

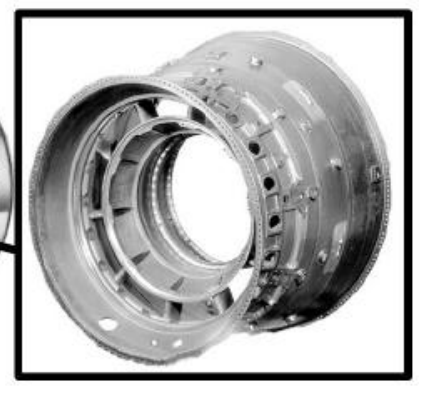

b

Fig. 1. Cutaway view of the V2500 aircraft engine with the diffuser assembly shown (a) and the actual diffuser assembly casting (b).

Problem Definition. The aerospace industry usually involves small series manufacturing, which requires certain finishing operations to be completed manually. Edge deburring of aerospace components with complex geometries is done by hand. The process is completed most often with dedicated filing tools or semi-automated by applying hand-held power cutters with small rotating blades. Aerospace engine components are usually exposed to high operating stress and vibration levels [19]. Burrs and sharp edges may reduce the mating performance of aerospace assembly components. It is then necessary to deburr and bevel or chamfer these edges. Aerospace component engineering involves strict acceptance criteria for finished parts to ensure high quality of production and operation. These stringent quality criteria provide the benefit of structural safety. It is not possible to assure the repeatability of precision machining processes without automation. While the human 
factor plays a major role in manufacturing processes, it can easily become their weakest link. Hand machining is required due to the presence of parts that are somewhat randomized in shape. This restricted randomization of shape is caused by the application of precision casting processes, the part geometry produced by which is inherently variable with the manufacturing precision of casting molds and solidification shrinkage. These effects cause problems with precise shape definition. This, in turn, results in the need for hand machining (such as edge deburring) and the impossibility of providing a repeatable definition of tool paths. This results in a high risk of producing parts with defects caused by human factors (such as fatigue, errors, or stress). It is extremely difficult to produce a strictly defined chamber along the entire processed edge by hand deburring. This has made it necessary to develop a new approach to the production of chamfers with specified quality characteristics.

Aircraft engine diffuser manufacturing requires the manual deburring of many edges. The V2500 engine diffuser assembly features ten types of edge forms that require deburring. The test detail was the three-cornered boss of the diffuser (Fig. 2). The as-cast surface condition of the three-cornered boss is shown in Fig. 2a. The machined surface and holes of the boss are shown in Fig. $2 b$.

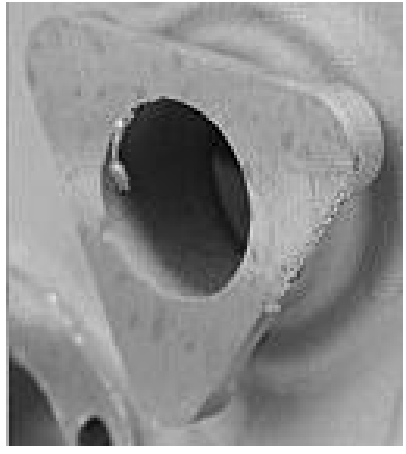

a

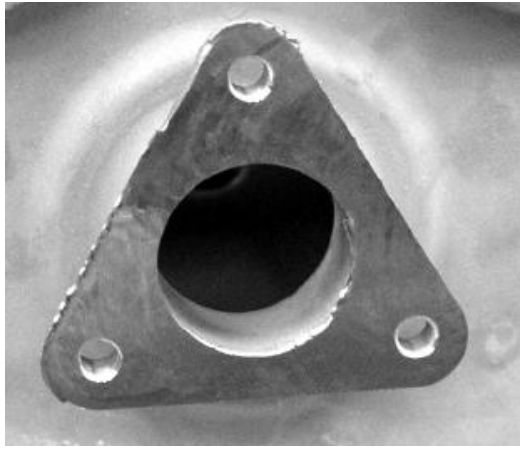

b

Fig. 2. The diffuser three-cornered boss to be machined: (a) as cast; (b) as machined with edges to deburr.

The edge deburring quality is visually inspected at several selected inspection points. In most deburring scenarios, the effects are visually inspected by a quality inspector. This quality inspection is intended to reject parts with sharp edges or notches. It is an undisputed fact that surface edges reduce the fatigue life of affected parts.

During high-speed machining, the non-stabilized pressure results in the impact of the tool edges with the workpiece. The high stresses in the tool invoke its local damages (Fig. 3a and b), which act as stress raisers. The material loss and damaged tool edge excite the tool vibration. The material processing by a damaged tool causes the irregular shape of the workpiece surface (Fig. 4). Each tool travel and workpiece processing cycle corresponds to the tool loading cycle. After some time, a complete failure of the tool occurs (Fig. 3c). An example of a damaged surface of a conical cutter and characteristic lines of the final fracture are depicted in Fig. 3d.

Methodology. Robotic system solutions may feature several manipulator trajectory adaptation methods, executed either in real time or according to predefined measurements. The manipulator trajectory adaptation to varying external conditions is determined by the process carried out with the manipulator. High-frequency processes with low contact forces feature proactive tools with variable contact force $[20,21]$. Odham [20] presented a method for deburring with a rotating wire brush. The key to the success of the method was the 


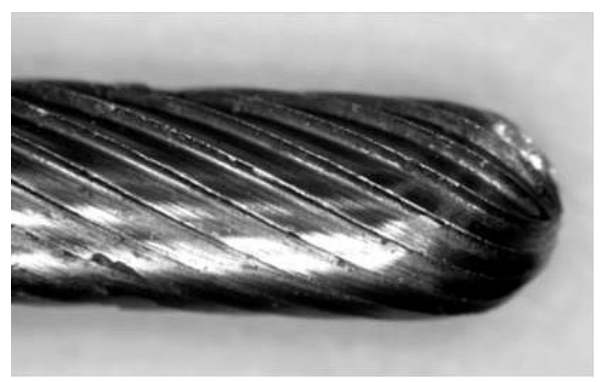

a

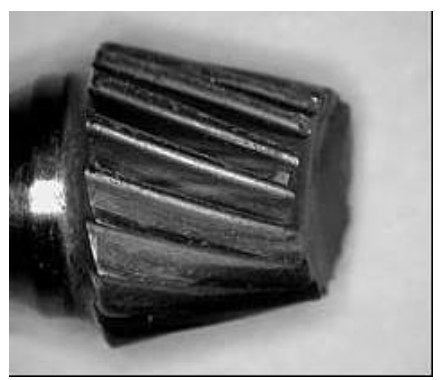

c

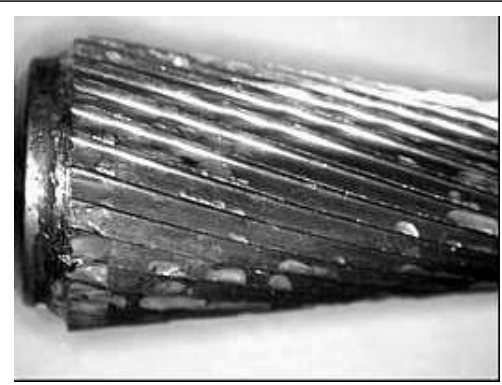

b

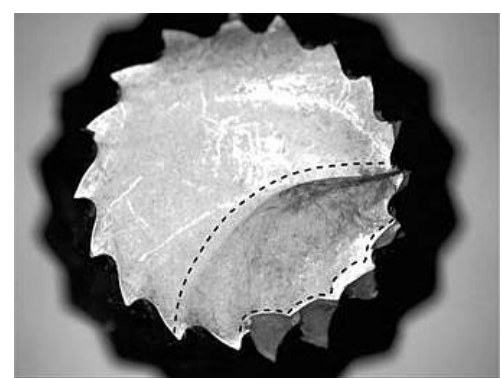

d

Fig. 3. Damaged zones obtained by treatment of machine parts from Inconel 718.
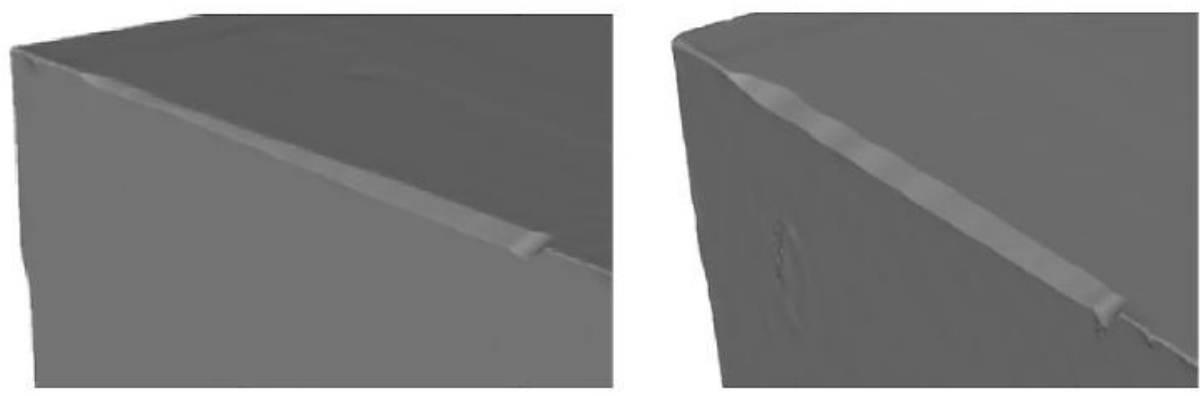

Fig. 4. 3D scans obtained after successive treatment iterations.

capacity of the robotic deburring system to deal with continuously variable part tolerances and burr sizes with active or passive force control tools. Shiakolas et al. [21] presented an application of a system based on parametric process modeling and an approach for solving the optimum process parameter definitions. Another work which presents an indirect force control strategy as a method for operating actual robotic controllers with advanced functionalities of torque force sensors was developed by Pires et al. [22]. A similar approach to this control process development was applied by Neto et al. [23].

The processes with contact forces of over $10 \mathrm{~N}$ use robotic force control systems [24, 25]. An alternative solution is the generation of the tool motion path by measuring the processed shape with laser or video sensor systems. Also noteworthy are learning systems. They include programming-coupled force control systems with software that records digitized tool trajectory points. In this case, the process is divided into the learning stage and the actual robot motion stage. Although very precise in performance, this type of solution requires much more time to execute. 
Approach. The developed manufacturing process was divided into three stages: addition (flash) removal, chamfering, and machined surface grinding. In the first stage, a dedicated tool with a support bearing is used to cut off the flash. This is done by a force controlled robot manipulator. In the second stage, the edges are burred with a pneumatic force progression tool. In the third stage, a force-controlled robot (Fig. 5a) performs the finish grinding of processed features.

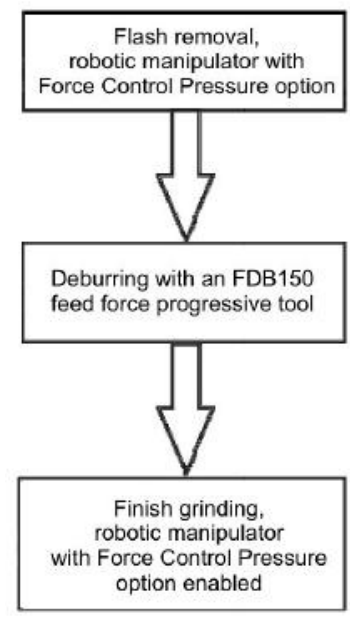

a

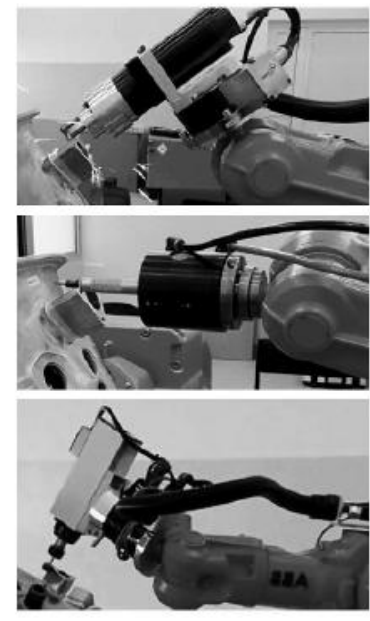

b

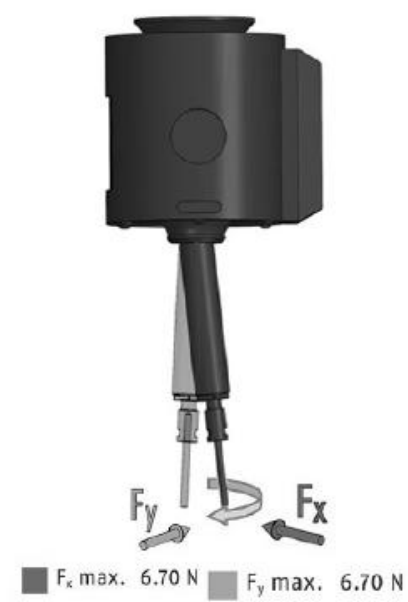

C

Fig. 5. Characteristics of the edge deburring solution: (a) process flowchart; (b) experimental process stage execution; (c) functional diagram of a rotary file with pneumatic feed force progression.

The entire experimental machining process discussed above was done with an ABB IRB140 robotic manipulator, whereas the successive stages were completed by retooling with a 4-position tool changer. The robot controller was digitally interfaced with a feed pressure setting system, which in turn was interfaced with the machining tool (FDB150). The pneumatic tool was programmed to operate (and control) the pressure between 1.4 and 4.1 bar. The initial experiments with the pneumatic tool helped to determine that it was not possible to effectively control pressure changes, i.e., the contact force. This was because of the complex geometrical shape of the diffuser details the machining of which was attempted. Given the relatively small amount of deducted material, the pneumatic tool was moving at a high velocity with low contact forces. The TCP motion velocity was high enough to result in a system feedback time value higher than the input change demand caused by the complex form of the workpiece. The compressibility of air in the pneumatic control system resulted in a tool response lag (an inertia of the system). This phenomenon attenuated high-frequency changes of the force preset by the robot's controller. This behavior necessitated the selection of machining parameters, or the definition of a motion execution strategy, air pressure values (and thus the contact force), and a TCP motion velocity.

Edge Deburring with Feed Force Progression. The implementation of pneumatic feed force progression of tools facilitated the machining of surfaces and edges; the locations (orientation and geometry) of which are unknown $[13,26]$. The machining process control with pneumatic feed force progression was insensitive to the movement of the threecornered boss edges. A general essential concept of this solution is shown in Fig. 5b, and the operating principle is shown in Fig. 5c. The allowed deviation of the tool axis from the tool base axis by $\pm 5 \mathrm{~mm}$ ensured insensitivity to the limited displacement of the machined surfaces. With the maximum spread of the as-cast three-cornered boss at $\pm 2 \mathrm{~mm}$, an offset 


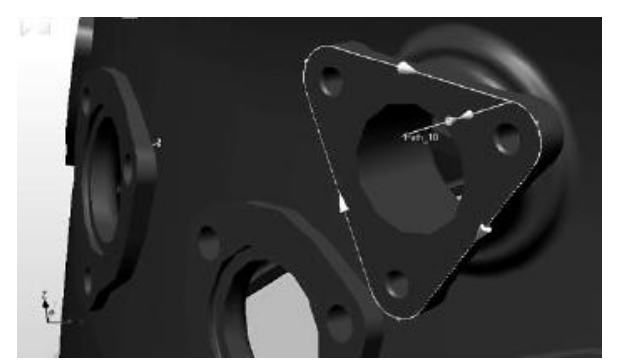

a

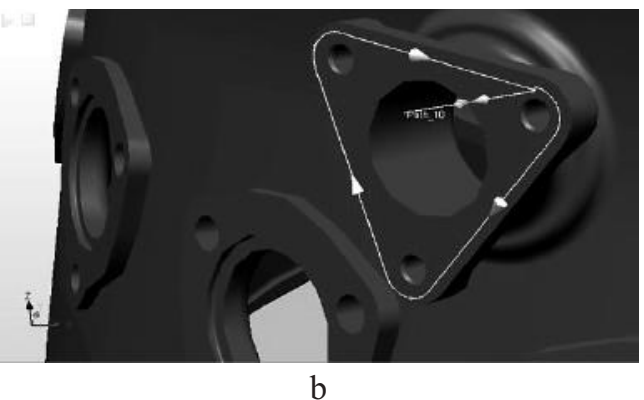

b

Fig. 6. RobotStudio machining process model: nominal (a) and offset (b) trajectories.

trajectory of the tool could be designed. The offset trajectory was determined with a nominal model, and the assumed offset trajectory value was doff $=1.7 \mathrm{~mm}$. Figure $6 \mathrm{a}$ shows an overview of the nominal model trajectory, whereas Fig. 6b shows the offset trajectory.

The critical parameters of the FDB150 machining tool were a rotational speed of $65,000 \mathrm{rpm}$ and a force adjustment range between 3.1 and $6.7 \mathrm{~N}$ (where the force was pressure-dependent).

A classical approach to robotic manipulator motion programming assumes that the workpiece is stationary in relation to the TCP trajectory. Here the angles at the robotic manipulator joints must be altered to execute a trajectory along complex geometric shapes. The more complex the shape is the higher the joint velocity and acceleration values that are needed. This results in the high imprecision of the preset TCP motion velocity. In machining conditions, this changes the deburring chamfer width, for example. To assure a smooth surface finish and the prevention of high material removal fluctuations, the robot manipulator work was executed with MultiMove interrelated motions. The MultiMove method is based on simultaneous movements of the workpiece and the TCP. This helped reduce the velocity change rates of robot manipulator joints (i.e., the translation of the positioning device helped to execute the trajectory); hence, the actual value of the TCP velocity was closer to the preset velocity value. Figure $7 \mathrm{a}$ shows the modeling of the three-cornered boss machining with MultiMove, and the actual execution of the modeling is shown in Fig. $7 \mathrm{~b}$.

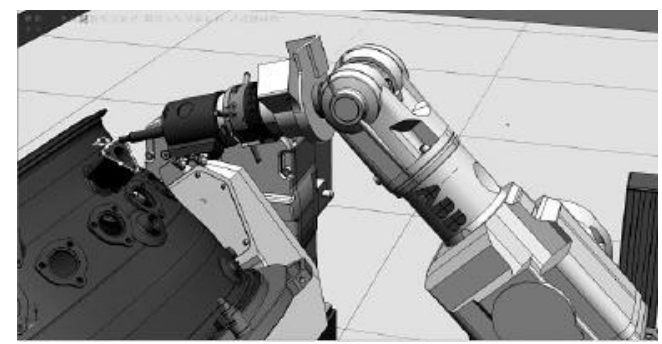

a

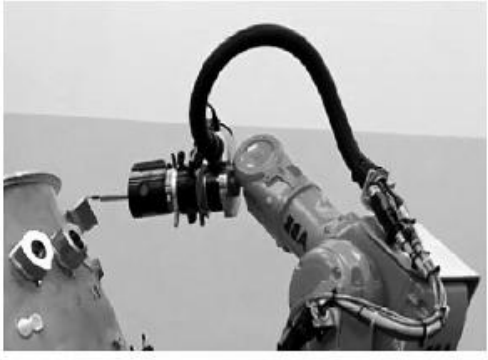

$\mathrm{b}$

Fig. 7. Execution of edge deburring with MultiMove: (a) as modeled in RobotStudio; (b) actual machining of the test workpiece.

Two cutting tool forms were studied at the initial stages of research: a slotting mill (HFC 0313.03.Z5 Lukas) and a tapered mill (HFM 0307.03.Z7). At later stages of research, the tapered mill was abandoned because of problems with the machining process stability, and especially the stability of the deburring chamfer width. 
Selection of Process Parameters. The series of tests was completed with robotic automatic measurement systems [27], due to the very large number of measurements required. The edge deburring process was controlled with a robotic workstation comprising an IRB1600 robot, a 3D scanner (Fig. 8), and the ATOS Professional software suite. The measurement control points were defined at locations with the highest expected deburring inaccuracy (Fig. 9a). Twelve such measurement control points were selected for the three-cornered boss, as shown in Fig. 9b (black line highlight). The chamfer deviations were analyzed with a $3 \mathrm{D}$ scan of the model at defined measurement control points. An example of a three-cornered boss scanned and converted to a 3D model is shown in Fig. 9c.

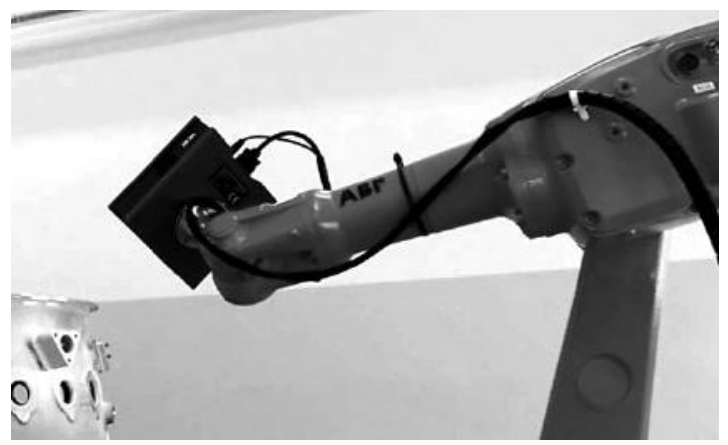

Fig. 8. The chamfer is measured with the $3 \mathrm{D}$ scanner.

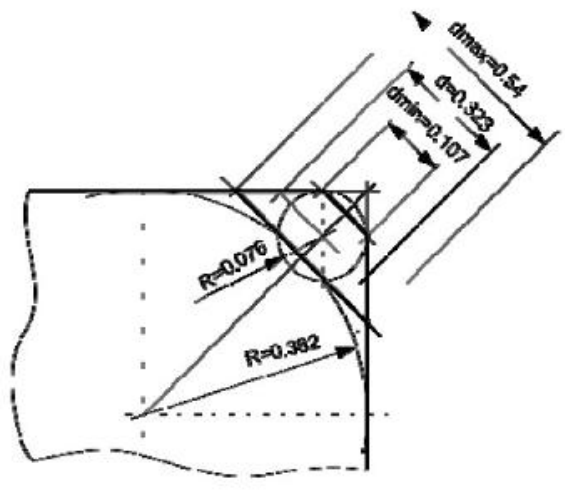

$\mathrm{a}$

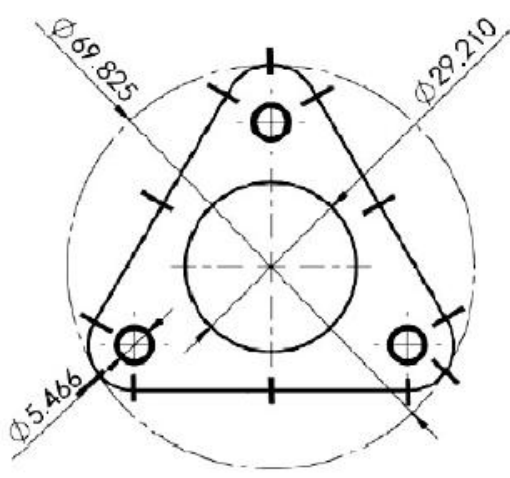

$\mathrm{b}$

Fig. 9. Characteristics of the chamfer width measurement method: (a) required deburring accuracy; (b) identification of the measurement control points.

Based on the required accuracy class, the deburring accuracy and deviation limits were defined for the nominal part detail. An experimental measurement was proposed to determine the produced chamfer width as a function of the contact force. The measurement results were plotted on a chart (Fig. 10) which represents the produced chamfer width values (Fig. 10a) and the tool feed force values (Fig. 10b). The measured chamfer width values were collated for the following robot TCP velocity values $V: 50,100,250,500,750$, and $1000 \mathrm{~mm} / \mathrm{s}$ (Fig. 11). The minimum and maximum limits of the chamfer width dimension were marked with continuous horizontal lines (Fig. 11b). The chart was plotted for the tool feed force values of 3.1, 4, 5, 6, and 6.7 N. An example of the measurement report for a single tool contact force and a single preset TCP velocity is shown in Fig. 11a. The chart in Fig. 11b was plotted with 12 measurement control points. There were 5 iterations of measurement at each measurement control point. 
A. Burghardt, D. Szybicki, K. Kurc, et al.

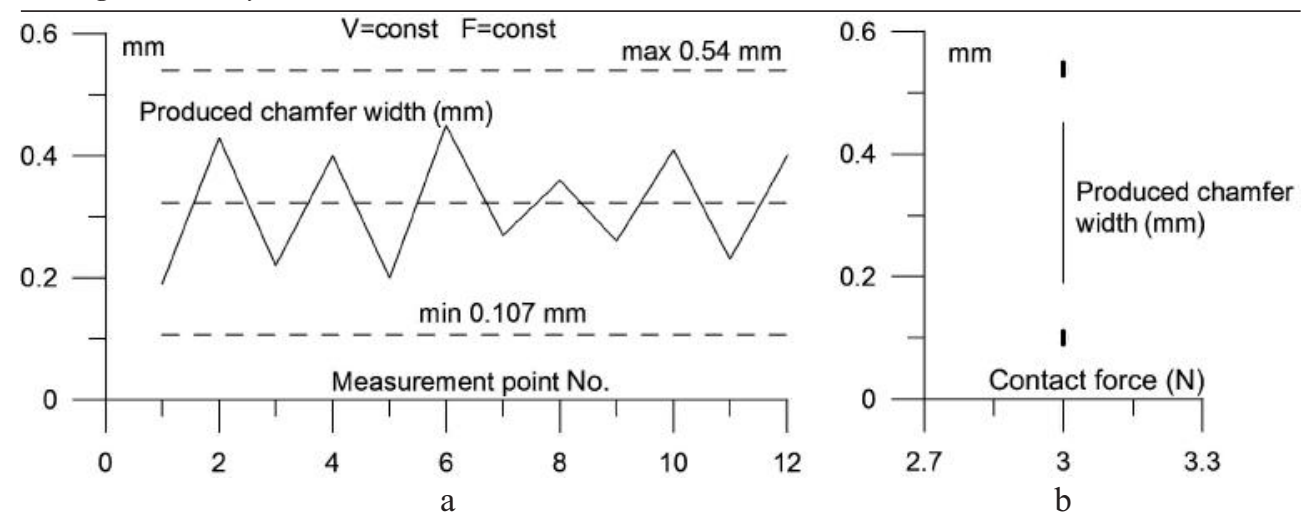

Fig. 10. Measurement results: (a) chamfer width values; (b) tool feed force.

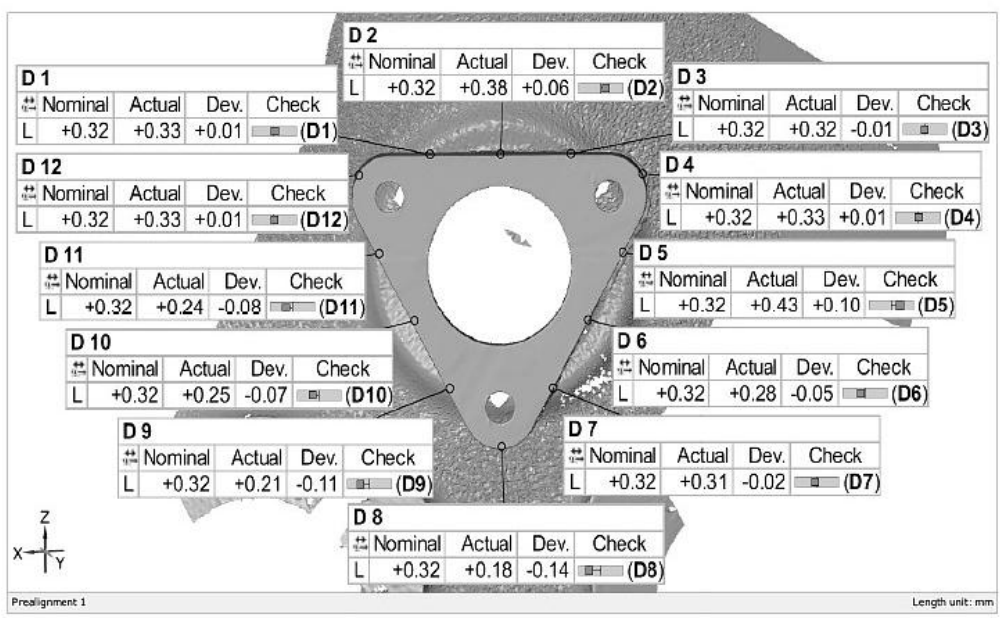

a

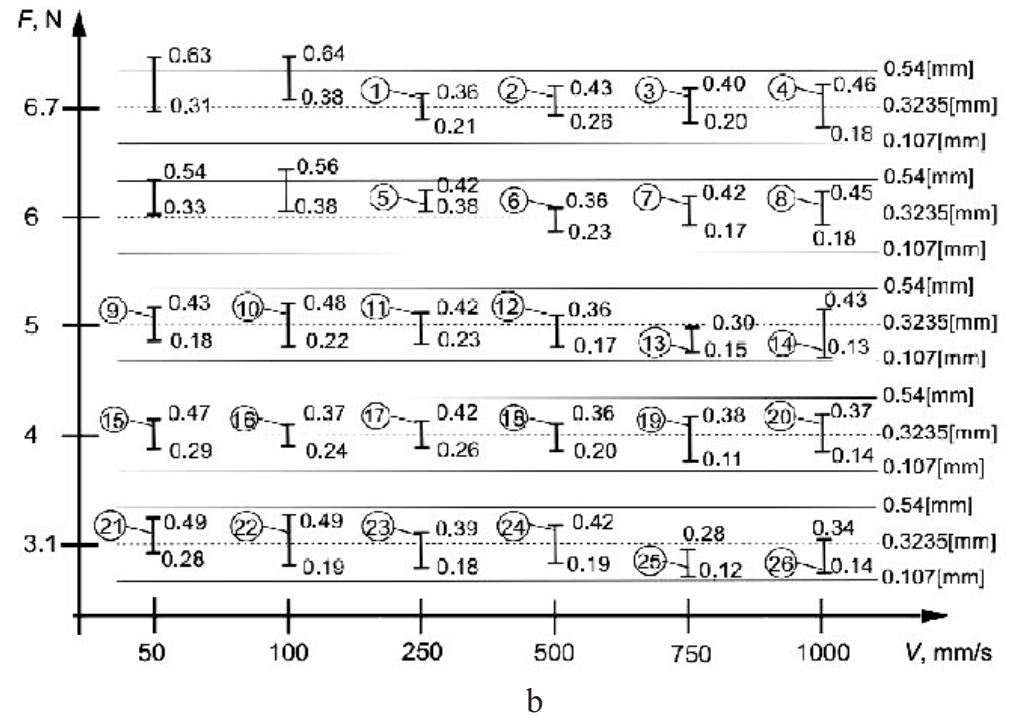

Fig. 11. Measurement results for the chamfer width (dimension): (a) example of a measurement report (point 9); (b) chamfer width (dimension) with limit values. 
Based on these results, the authors hereof noted that there was a wide span of the following process parameters at the nominal preset tool velocity $(V)$ : contact force and TCP motion velocity, at which the chamfer width meets the manufacturing specification requirements. An optimisation procedure was proposed to facilitate the selection of those parameters of the parameter set which ensure the best execution of the machining process in question.

Optimization of Process Parameters. The real-life quality control of deburring at manufacturing plants is done by visual inspection. This permits deburring chamfer width values within a predefined tolerance range; however, the values themselves may vary greatly within the tolerance limits (with sudden changes in width). A deburring chamfer with those characteristics is unacceptable.

As a result of the experimental research, 26 two-element parameter sets were produced (Fig. 11b) which ensured machining in accordance with the predefined requirements. An optimization procedure was proposed to select a suboptimal set of parameters. First, the parameters were organized in an ascending order with a quality indicator assumed for the research. The quality indicator was the sum square of chamfer nominal width value deviations. The maximum shape change function differential coefficient value was used as an indicator to determine the surface shape change rate. The nominal width value deviation was digitized into a set of points: the tool path was divided by $n$ points spaced every $1 \mathrm{~mm}$, and the chamfer width error values that corresponded to these points were recorded with reference to the nominal width value (level 0 in Fig. 12).

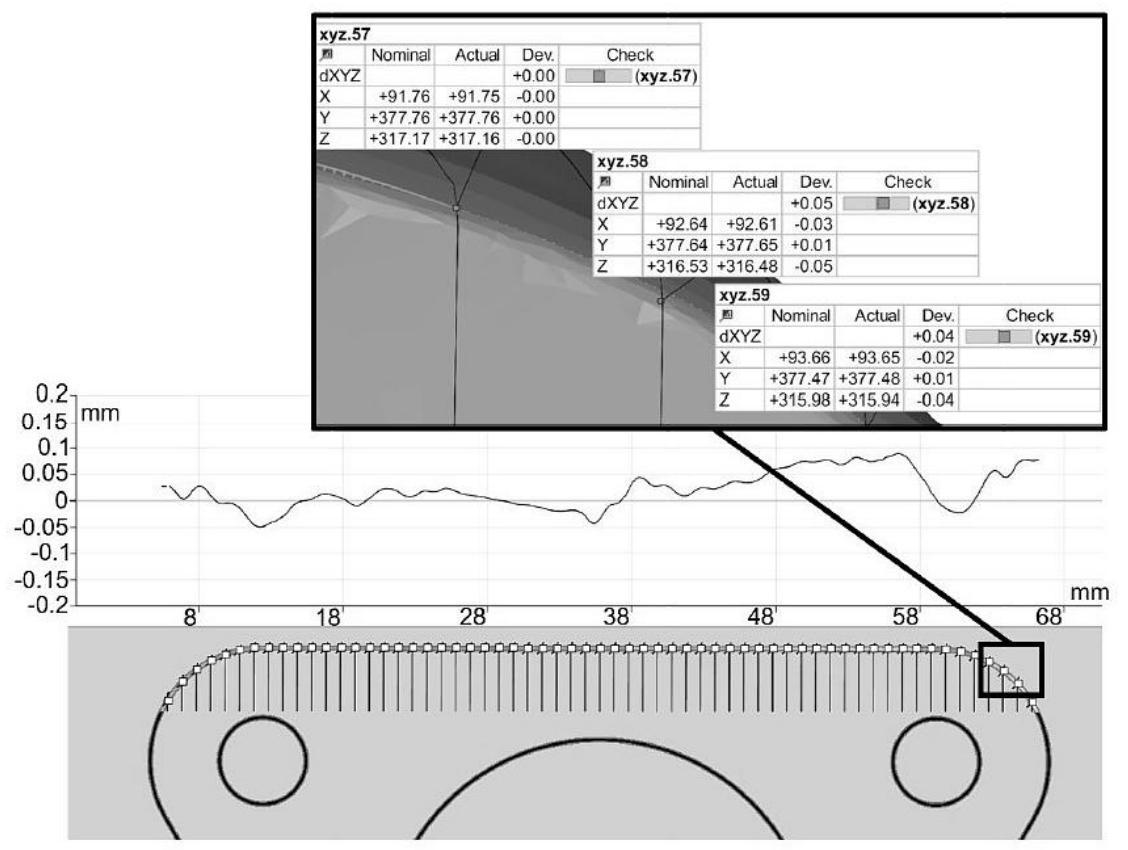

Fig. 12. Produced chamfer accuracy changes vs. tool path. (Three-cornered boss top edge, measurement No. 16.)

The resulting values at the points were then approximated with a 3rd order spline in Matlab/Simulink:

$$
F(n)=e,
$$

where $e$ is the chamfer width value error and $n$ the respective tool path point. 

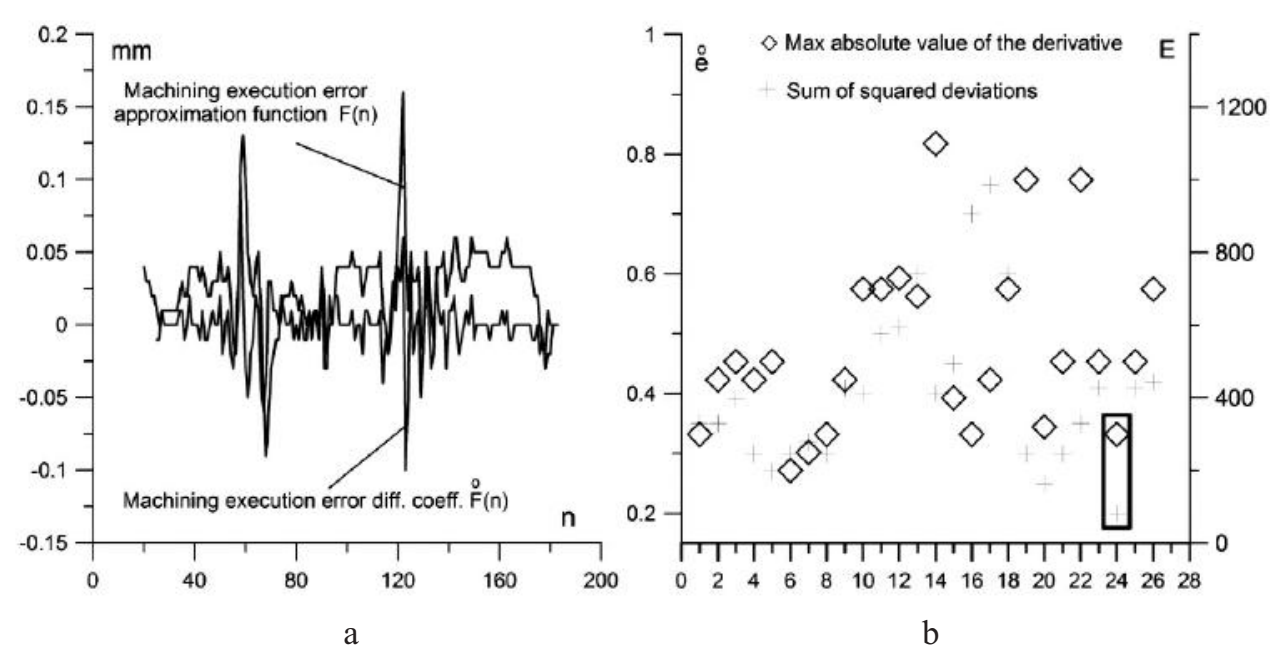

Fig. 13. Function of machining execution accuracy value changes and its differential coefficient for measurement No. 16 (a) and list of quality indicators (b).

Second, the generated function was used to determine the proposed quality indicators for the measurements at points No. 1 to 26 (Fig. 11b). The quality indicators corresponded to a sum square of the nominal width value deviation, and the maximum absolute value of the error function differential coefficient (Fig. 13a). The sum square indicator was determined for every measurement from:

$$
E=\int_{0}^{N} e^{2} d n
$$

whereas for the error function differential coefficient maximum absolute value:

$$
\dot{e}_{\max }=\max _{n \in N}\left(\left|e_{n}\right|\right) .
$$

Collating the resulting indicator values in Fig. 13b helped select the process parameters from measurement No. 24. The resulting parameters of tool contact force and TCP velocity at the preset nominal tool rotational speed were adopted as the best solution to the available set.

Conclusions. The experimental results presented herein provide the tolerance limits of compliant tools that can be applied to effective edge deburring. Note that the results were obtained for Inconel 718 and an HFC 0313.03.25 machining tool, and as such cannot be universally applied. The authors advocate a procedure of work that facilitates the selection of suboptimal process parameters. In the case contemplated herein, the suboptimal process parameters were the TCP velocity and the tool contact force.

The proposed optimization procedure helped to indicate a set of process parameters that ensured machining in conformity with the process specifications. The solution is an engineering one by nature and not a classic case of finding a function extremum with the two adopted criteria (the quality indicators discussed above). An advantage of the proposed solution lies in its simplicity which ensures the improvement of edge deburring quality. This is very important for industrial applications. To recapitulate, the best processing values are: tool rotational speed $=65,000 \mathrm{rpm}$, tool contact force $=3.1 \mathrm{~N}$, and TCP velocity $=$ $500 \mathrm{~mm} / \mathrm{s}$. 
1. N. Mendes, P. Neto, J. N. Pires, and A. P. Moreira, "Fuzzy-PI force control for industrial robotics," in: P. Vadakkepat, J. H. Kim, N. Jesse, et al. (Eds.), Trends in Intelligent Robotics (Proc. of the 13th FIRA Robot World Congress, FIRA 2010, September 15-17, 2010, Bangalore, India), Springer, Heidelberg (2010), pp. 322329.

2. N. Mendes, P. Neto, J. N. Pires, and A. Loureiro, "An optimal fuzzy-PI force/motion controller to increase industrial robot autonomy," Int. J. Adv. Manuf. Tech., 68, 435-441 (2013).

3. A. Lopes and F. Almeida, "A force-impedance controlled industrial robot using an active robotic auxiliary device," Robot. Comput. Integr. Manuf., 24, No. 3, 299-309 (2008).

4. G. C. Vosniakos and E. Matsas, "Improving feasibility of robotic milling through robot placement optimization," Robot. Comput. Integr. Manuf., 26, No. 5, 517-525 (2010).

5. P. Gierlak, "Hybrid position/force control in robotised machining," Solid State Phenom.," 210, 192-199 (2014).

6. P. Gierlak, "Hybrid position/force control of the SCORBOT-ER 4pc manipulator with neural compensation of nonlinearities," in: L. Rutkowski, M. Korytkowski, R. Scherer, et al. (Eds.), Artificial Intelligence and Soft Computing (Proc. of the 11th Int. Conf. ICAISA 2012, April 29 - May 3, 2012, Zakopane, Poland), Part II, LNCS 7268, Springer, Berlin-Heidelberg (2012), pp. 433-441.

7. A. Burghardt, D. Szybicki, K. Kurc, and M. Muszyńska, "Optimization of process parameters of edge robotic deburring with force control," Int. J. Appl. Mech. Eng., 21, No. 4, 987-995 (2016).

8. U. Zuperl, F. Cus, and M. Milfelner, "Fuzzy control strategy for an adaptive force control in end-milling," J. Mater. Process. Tech., 164-165, 1472-1478 (2005).

9. J. Z. Zhang, J. C. Chen, and E. D. Kirby, "Surface roughness optimization in an end-milling operation using the Taguchi design method," J. Mater. Process. Tech., 184, 233-239 (2007).

10. S. P. Khanghah, M, Boozarpoor, M. Lotfi, and R. Teimouri, "Optimization of micro-milling parameters regarding burr size minimization via RSM and simulated annealing algorithm," T. Indian I. Metals, 68, No. 5, 897-910 (2015).

11. R. S. Jamisola, Jr., D. N. Oetomo, M. H. Ang, Jr., et al., "Compliant motion using a mobile manipulator: an operational space formulation approach to aircraft canopy polishing," Adv. Robotics, 19, No. 5, 613-634 (2005).

12. P. Gierlak, A. Burghardt, D. Szybicki, et al., "On-line manipulator tool condition monitoring based on vibration analysis," Mech. Syst. Signal Pr., 89, 14-26 (2016).

13. A. Burghardt, K. Kurc, M. Muszyńska, and D. Szybicki, "Robotic station with force control," Modelowanie Inżynierskie, 22, No. 53, 30-36 (2014).

14. Z. Doulgeri and Y. Karayiannidis, "An adaptive force regulator for a robot in compliant contact with an unknown surface," in: Proc. of the 2005 IEEE Int. Conf. on Robotics and Automation (April 18-22, 2005, Barcelona), IEEE (2005), pp. 2685- 2690.

15. Z. Doulgeri and Y. Karayiannidis, "Force/position tracking of a robot in compliant contact with unknown stiffness and surface kinematics," in: Proc. of the 2007 IEEE Int. Conf. on Robotics and Automation (April 10-14, 2007, Roma), IEEE (2007), pp. 4190-4195.

16. Z. Deng, H. Jin, Y. Hu, et al., "Fuzzy force control and state detection in vertebral lamina milling," Mechatronics, 35, 1-10 (2016). 
17. S. Matsuoka, K. Shimizu, N. Yamazaki, and Y. Oki, "High-speed end milling of an articulated robot and its characteristics," J. Mater. Process. Tech., 95, 83-89 (1999).

18. E. Abele, M. Weigold, and S. Rothenbücher, "Modeling and identification of an industrial robot for machining applications," CIRP Annals, 56, No. 1, 387-390 (2007).

19. N. Jayaweera and P. Webb, "Measurement assisted robotic edge deburring of aero engine components," WSEAS Trans. Syst. Control, 5, No. 3, 174-183 (2010).

20. A. Odham, "Successful robotic deburring is really a matter of choices," Tool. Prod., 73, No. 12, 14-19 (2007).

21. P. S. Shiakolas, D. Labalo, J. M. Fitzgerald, "RobSurf: A near real time OLP system for robotic surface finishing," in: Proc. of the 7th Mediterranean Conf. on Control and Automation (MED99) (June 28-30, 1999, Haifa, Israel), http://med.ee.nd.edu/MED7/ med99/papers/MED128.pdf.

22. J. N. Pires, G. Afonso, and N. Estrela, "Force control experiments for industrial applications: a test case using an industrial deburring example," Assembly Autom., 27, No. 2, 148-156 (2007).

23. P. Neto, N. Mendes, J. N. Pires, and A. P. Moreira, "CAD-based robot programming: The role of Fuzzy-PI force control in unstructured environments," in: Proc. of the 2010 IEEE Int. Conf. on Automation Science and Engineering (August 21-24, 2010, Toronto), IEEE (2010), pp. 362-367.

24. A. Burghardt, K. Kurc, and D. Szybicki, "Robotic automation of the turbo-propeller engine blade grinding process," Appl. Mech. Mater., 817, 206-213 (2016).

25. Z. Hendzel, A. Burghardt, P. Gierlak, and M. Szuster, "Conventional and fuzzy force control in robotised machining," Sol. St. Phen., 210, 178-185 (2014).

26. P. Zhao and Y. Shi, "Posture adaptive control of the flexible grinding head for blisk manufacturing," Int. J. Adv. Manuf. Tech., 70, 1989-2001 (2014).

27. A. Burghardt, K. Kurc, M. Muszyńska, and D. Szybicki, Robotic position to verify the machining process, Modelowanie Inżynierskie, 21, No. 52, 23-29 (2014). 